Article

\title{
Some Identities and Inequalities Involving Symmetry Sums of Legendre Polynomials
}

\section{Tingting Wang * and Liang Qiao}

College of Science, Northwest A\&F University, Yangling 712100, China; 18309225762@163.com

* Correspondence: ttwang@nwsuaf.edu.cn

Received: 13 November 2019; Accepted: 12 December 2019; Published: 16 December 2019

check for updates

Abstract: By using the analysis methods and the properties of Chebyshev polynomials of the first kind, this paper studies certain symmetry sums of the Legendre polynomials, and gives some new and interesting identities and inequalities for them, thus improving certain existing results.

Keywords: Legendre polynomials; Chebyshev polynomials of the first kind; power series; symmetry sums; polynomial identities; polynomial inequalities

\section{Introduction}

For any integer $n \geq 0$, the Legendre polynomials $\left\{P_{n}(x)\right\}$ are defined as follows:

$$
P_{n}(x)=\frac{2 n-1}{n} x P_{n-1}(x)-\frac{n-1}{n} P_{n-2}(x)
$$

for all $n \geq 2$, with $P_{0}(x)=1$ and $P_{1}(x)=x$, see [1,2] for more information.

The first few terms of $P_{n}(x)$ are $P_{2}(x)=\frac{1}{2}\left(3 x^{2}-1\right), P_{3}(x)=\frac{1}{2}\left(5 x^{3}-3 x\right)$, $P_{4}(x)=\frac{1}{8}\left(35 x^{4}-30 x^{2}+3\right), P_{5}(x)=\frac{1}{8}\left(63 x^{5}-70 x^{3}+15 x\right), \cdots$.

In fact, the general term of $P_{n}(x)$ is given by the formula

$$
P_{n}(x)=\frac{1}{2^{n}} \cdot \sum_{k=0}^{\left[\frac{n}{2}\right]} \frac{(-1)^{k} \cdot(2 n-2 k) !}{k ! \cdot(n-k) ! \cdot(n-2 k) !} \cdot x^{n-2 k}
$$

where $[y]$ denotes the greatest integer less than or equal to $y$.

It is clear that $P_{n}(x)$ is an orthogonal polynomial (see [1,2]). That is,

$$
\int_{-1}^{1} P_{m}(x) P_{n}(x) d x= \begin{cases}0, & \text { if } m \neq n ; \\ \frac{2}{2 n+1}, & \text { if } m=n .\end{cases}
$$

The generating function of $P_{n}(x)$ is

$$
\frac{1}{\sqrt{1-2 x t+t^{2}}}=\sum_{n=0}^{\infty} P_{n}(x) \cdot t^{n},|x| \leq 1,|t|<1 .
$$

These polynomials play a vital role in the study of function orthogonality and approximation theory, as a result, some scholars have dedicated themselves to studying their various natures and obtained a series of meaningful research results. The studies that are concerned with this content can be found in [1-20]. Recently, Shen Shimeng and Chen Li [3] give certain symmetry sums of $P_{n}(x)$, and proved the following result: 
For any positive integer $k$ and integer $n \geq 0$, one has the identity

$$
\begin{aligned}
& (2 k-1) ! ! \sum_{a_{1}+a_{2}+\cdots+a_{2 k+1}=n} P_{a_{1}}(x) P_{a_{2}}(x) \cdots P_{a_{2 k+1}}(x) \\
= & \sum_{j=1}^{k} C(k, j) \sum_{i=0}^{n} \frac{(n+k+1-i-j) !}{(n-i) !} \cdot \frac{\left(\begin{array}{c}
i+j+k-2 \\
i
\end{array}\right)}{x^{k-1+i+j}} \cdot P_{n+k+1-i-j}(x),
\end{aligned}
$$

where $(2 k-1) ! !=(2 k-1) \cdot(2 k-3) \cdots 3 \cdot 1$, and $C(k, i)$ is a recurrence sequence defined by $C(k, 1)=1$, $C(k+1, k+1)=(2 k-1) !$ ! and $C(k+1, i+1)=C(k, i+1)+(k-1+i) \cdot C(k, i)$ for all $1 \leq i \leq k-1$.

The calculation formula for the sum of Legendre polynomials given above is virtually a linear combination of some $P_{n}(x)$, and the coefficients $C(k, i)$ are very regular. However, the result is in the form of a recursive formula, in other words, especially when $k$ is relatively large, the formula is not actually easy to use for calculating specific values.

In an early paper, Zhou Yalan and Wang Xia [4] obtained some special cases with $k=3$ and $k=5$. It is even harder to calculate their exact values for the general positive integer $k$, especially if $k$ is large enough.

Naturally, we want to ask a question: Is there a more concise and specific formula for the calculation of the above problems? This is the starting point of this paper. We used the different methods to come up with additional simpler identities. It is equal to saying that we have used the analysis method and the properties of the first kind of Chebyshev polynomials, thereby establishing the symmetry of the Legendre polynomial and symmetry relationship with the first kind of Chebyshev polynomial, and proved the following three results:

Theorem 1. For any integers $k \geq 1$ and $n \geq 0$, we have the identity

$$
\sum_{a_{1}+a_{2}+\cdots+a_{k}=n} P_{a_{1}}(x) \cdot P_{a_{2}}(x) \cdots P_{a_{k}}(x)=\sum_{i=0}^{n} \frac{<\frac{k}{2}>_{i}}{i !} \cdot \frac{<\frac{k}{2}>_{n-i}}{(n-i) !} \cdot T_{n-2 i}(x),
$$

where $<x>_{0}=1,<x>_{k}=x(x+1)(x+2) \cdots(x+k-1)$ for all integers $k \geq 1$, and $T_{n}(x)=T_{-n}(x)=$ $\frac{1}{2}\left(\left(x+\sqrt{x^{2}-1}\right)^{n}+\left(x+\sqrt{x^{2}-1}\right)^{n}\right)$ denotes Chebyshev polynomials of the first kind.

Theorem 2. Let $q>1$ is an integer, $\chi$ is any primitive character mod $q$. Then for any integers $k \geq 1$ and $n \geq 0$, we have the inequality

$$
\begin{aligned}
& \left|\sum_{a_{1}+a_{2}+\cdots+a_{k}=n} \sum_{a=1}^{q} \chi(a) P_{a_{1}}\left(\cos \frac{2 \pi a}{q}\right) \cdot P_{a_{2}}\left(\cos \frac{2 \pi a}{q}\right) \cdots P_{a_{k}}\left(\cos \frac{2 \pi a}{q}\right)\right| \\
& \leq \sqrt{q} \cdot\left(\begin{array}{c}
n+k-1 \\
k-1
\end{array}\right) .
\end{aligned}
$$

Theorem 3. For any integer $n \geq 0$ with $2 \nmid n$, we have the identity

$$
\begin{aligned}
& \int_{-\frac{\pi}{2}}^{\frac{\pi}{2}}\left(\sum_{a_{1}+a_{2}+\cdots+a_{k}=n} P_{a_{1}}(\sin \theta) \cdot P_{a_{2}}(\sin \theta) \cdots P_{a_{k}}(\sin \theta)\right)^{2} d \theta \\
= & 2 \pi \cdot \sum_{i=0}^{\left[\frac{n}{2}\right]}\left(\frac{<\frac{k}{2}>_{i}}{i !} \cdot \frac{<\frac{k}{2}>_{n-i}}{(n-i) !}\right)^{2} ;
\end{aligned}
$$

If $n=2 m$, then we have 


$$
\begin{aligned}
& \int_{-\frac{\pi}{2}}^{\frac{\pi}{2}}\left(\sum_{a_{1}+a_{2}+\cdots+a_{k}=n} P_{a_{1}}(\sin \theta) \cdot P_{a_{2}}(\sin \theta) \cdots P_{a_{k}}(\sin \theta)\right)^{2} d \theta \\
= & 2 \pi \cdot \sum_{i=0}^{m}\left(\frac{<\frac{k}{2}>_{i}}{i !} \cdot \frac{<\frac{k}{2}>_{2 m-i}}{(2 m-i) !}\right)^{2}-\pi \cdot\left(\frac{<\frac{k}{2}>_{m}}{m !}\right)^{4} .
\end{aligned}
$$

Essentially, the main result of this paper is Theorem 1, which not only reveals the profound properties of Legendre polynomials and Chebyshev polynomials, but also greatly simplifies the calculation of the symmetry sum of Legendre polynomials in practice. We can replace the calculation of the symmetric sum of the Legendre polynomial with the first single Chebyshev polynomial calculation, which can greatly simplify the calculation of the symmetric sum.

Theorem 2 gives an upper bound estimate of the character sum of Legendre polynomials. Theorem 3 reveals the orthogonality of the symmetry sum of Legendre polynomials, which is a generalization of the orthogonality of functions. Of course, Theorems 2 and 3 can also be seen as the direct application of Theorem 1 in analytical number theory and the orthogonality of functions. This is of great significance in analytic number theory, and it has also made new contributions to the study of Gaussian sums.

In fact if we taking $k=1$, and note that the identity $\frac{<\frac{1}{2}>h}{h !}=\frac{1}{4^{h}} \cdot\left(\begin{array}{c}2 h \\ h\end{array}\right)$, then from our theorems we may immediately deduce the following three corollaries.

Corollary 1. For any integer $n \geq 0$, we have the identity

$$
P_{n}(x)=\frac{1}{4^{n}} \sum_{i=0}^{n}\left(\begin{array}{c}
2 i \\
i
\end{array}\right)\left(\begin{array}{c}
2 n-2 i \\
n-i
\end{array}\right) \cdot T_{n-2 i}(x),
$$

where $T_{n}(x)$ denotes Chebyshev polynomials of the first kind.

Corollary 2. Let $q>1$ is an integer, $\chi$ is any primitive character $\bmod q$. Then for any integer $n \geq 0$, we have the inequality

$$
\left|\sum_{a=1}^{q} \chi(a) P_{n}\left(\cos \frac{2 \pi a}{q}\right)\right| \leq \sqrt{q} .
$$

Corollary 3. For any integer $n \geq 0$ with $2 \nmid n$, we have the identity

$$
\int_{-\frac{\pi}{2}}^{\frac{\pi}{2}} P_{n}^{2}(\sin \theta) d \theta=\frac{2 \pi}{4^{2 n}} \sum_{i=0}^{\left[\frac{n}{2}\right]}\left(\begin{array}{c}
2 i \\
i
\end{array}\right)^{2}\left(\begin{array}{c}
2 n-2 i \\
n-i
\end{array}\right)^{2}
$$

If $n=2 m$, then we have the identity

$$
\int_{-\frac{\pi}{2}}^{\frac{\pi}{2}} P_{n}^{2}(\sin \theta) d \theta=\frac{2 \pi}{4^{2 n}} \sum_{i=0}^{\left[\frac{n}{2}\right]}\left(\begin{array}{c}
2 i \\
i
\end{array}\right)^{2}\left(\begin{array}{c}
2 n-2 i \\
n-i
\end{array}\right)^{2}-\frac{\pi}{4^{2 n}} \cdot\left(\begin{array}{c}
2 m \\
m
\end{array}\right)^{4} .
$$

\section{Proofs of the Theorems}

In this section, we will directly prove the main results in this paper by by means of the properties of characteristic roots.

Proof of Theorem 1. First we prove Theorem 1. Let $\alpha=x+\sqrt{x^{2}-1}$ and $\beta=x-\sqrt{x^{2}-1}$ be two characteristic roots of the characteristic equation $\lambda^{2}-2 x \lambda+1=0$. Then from the definition and properties of Chebyshev polynomials $T_{n}(x)$ of the first kind, we have 


$$
T_{n}(x)=T_{-n}(x)=\frac{1}{2}\left(\alpha^{n}+\beta^{n}\right), n \geq 0 .
$$

For any positive integer $k$, combining properties of power series and Formula (1) we have the identity

$$
\begin{aligned}
& \left(\frac{1}{\sqrt{1-2 x t+t^{2}}}\right)^{k}=\frac{1}{\left(1-2 x t+t^{2}\right)^{\frac{k}{2}}}=\frac{1}{(1-\alpha t)^{\frac{k}{2}}(1-\beta t)^{\frac{k}{2}}} \\
& =\sum_{n=0}^{\infty}\left(\sum_{a_{1}+a_{2}+\cdots+a_{k}=n} P_{a_{1}}(x) \cdot P_{a_{2}}(x) \cdot P_{a_{3}}(x) \cdots P_{a_{k}}(x)\right) \cdot t^{n} .
\end{aligned}
$$

At the same time, we focus on the power series

$$
\frac{1}{(1-x)^{\frac{k}{2}}}=\sum_{n=0}^{\infty} \frac{<\frac{k}{2}>_{n}}{n !} \cdot x^{n},|x|<1,
$$

where $<x>_{0}=1,<x>_{h}=x(x+1)(x+2) \cdots(x+h-1)$ for all integers $h \geq 1$.

So for any positive integer $k$, note that $\alpha \cdot \beta=1$, from (3) and the symmetry properties of $\alpha$ and $\beta$ we have

$$
\begin{aligned}
& \left(\frac{1}{\sqrt{1-2 x t+t^{2}}}\right)^{k}=\frac{1}{(1-\alpha t)^{\frac{k}{2}}(1-\beta t)^{\frac{k}{2}}} \\
& =\left(\sum_{n=0}^{\infty} \frac{<\frac{k}{2}>_{n}}{n !} \cdot \alpha^{n} \cdot t^{n}\right)\left(\sum_{n=0}^{\infty} \frac{<\frac{k}{2}>_{n}}{n !} \cdot \beta^{n} \cdot t^{n}\right) \\
& =\sum_{n=0}^{\infty}\left(\sum_{i=0}^{n} \frac{<\frac{k}{2}>_{i}}{i !} \cdot \frac{<\frac{k}{2}>_{n-i}}{(n-i) !} \cdot \alpha^{i} \cdot \beta^{n-i}\right) \cdot t^{n} \\
& =\sum_{n=0}^{\infty}\left(\sum_{i=0}^{n} \frac{<\frac{k}{2}>_{i}}{i !} \cdot \frac{<\frac{k}{2}>_{n-i}}{(n-i) !} \cdot \beta^{n-2 i}\right) \cdot t^{n} \\
& =\sum_{n=0}^{\infty}\left(\sum_{i=0}^{n} \frac{<\frac{k}{2}>_{i}}{i !} \cdot \frac{<\frac{k}{2}>_{n-i}}{(n-i) !} \cdot \alpha^{n-2 i}\right) \cdot t^{n} \\
& =\sum_{n=0}^{\infty}\left(\sum_{i=0}^{n} \frac{<\frac{k}{2}>_{i}}{i !} \cdot \frac{<\frac{k}{2}>_{n-i}}{(n-i) !} \cdot \frac{1}{2}\left(\alpha^{n-2 i}+\beta^{n-2 i}\right)\right) \cdot t^{n} \\
& =\sum_{n=0}^{\infty}\left(\sum_{i=0}^{n} \frac{<\frac{k}{2}>_{i}}{i !} \cdot \frac{<\frac{k}{2}>_{n-i}}{(n-i) !} \cdot T_{n-2 i}(x)\right) \cdot t^{n} .
\end{aligned}
$$

Combining (2) and (4), and then by comparing the coefficients on both sides of the power series, we can find

$$
\sum_{a_{1}+a_{2}+\cdots+a_{k}=n} P_{a_{1}}(x) \cdot P_{a_{2}}(x) \cdots P_{a_{k}}(x)=\sum_{i=0}^{n} \frac{<\frac{k}{2}>_{i}}{i !} \cdot \frac{<\frac{k}{2}>_{n-i}}{(n-i) !} \cdot T_{n-2 i}(x) .
$$

This proves Theorem 1.

Proof of Theorem 2. The proof of Theorem 2 is next. Let $q>1$ be any integer, $\chi$ denotes any primitive character $\bmod q$. Then from Theorem 1 with $x=\cos \left(\frac{2 \pi a}{q}\right)$ and the identity $T_{n}(\cos \theta)=\cos (n \theta)$, we have 


$$
\begin{aligned}
& \sum_{a_{1}+a_{2}+\cdots+a_{k}=n} \sum_{a=1}^{q} \chi(a) P_{a_{1}}\left(\cos \frac{2 \pi a}{q}\right) \cdot P_{a_{2}}\left(\cos \frac{2 \pi a}{q}\right) \cdots P_{a_{k}}\left(\cos \frac{2 \pi a}{q}\right) \\
= & \sum_{i=0}^{n} \frac{<\frac{k}{2}>_{i}}{i !} \cdot \frac{<\frac{k}{2}>_{n-i}}{(n-i) !} \cdot \sum_{a=1}^{q} \chi(a) \cdot \cos \left(\frac{2 \pi a(n-2 i)}{q}\right) \\
= & \frac{1}{2} \sum_{i=0}^{n} \frac{<\frac{k}{2}>_{i}}{i !} \cdot \frac{<\frac{k}{2}>_{n-i}}{(n-i) !} \cdot \sum_{a=1}^{q} \chi(a)\left(e\left(\frac{a(n-2 i)}{q}\right)+e\left(\frac{-a(n-2 i)}{q}\right)\right) \\
= & \frac{\tau(\chi)}{2} \sum_{i=0}^{n} \frac{<\frac{k}{2}>_{i}}{i !} \cdot \frac{<\frac{k}{2}>_{n-i}}{(n-i) !} \cdot(\bar{\chi}(n-2 i)+\bar{\chi}(-(n-2 i))),
\end{aligned}
$$

where $e(y)=e^{2 \pi i y}$, and $\sum_{a=1}^{q} \chi(a) e\left(\frac{n a}{q}\right)=\bar{\chi}(n) \tau(\chi)$.

Note that for any primitive character $\chi \bmod q$, from the properties of Gauss sums, we have $|\tau(\chi)|=\sqrt{q}$, and for any positive integer $k \geq 1$, we have

$$
\begin{aligned}
& \frac{1}{(1-x)^{k}}=\sum_{n=0}^{\infty}\left(\begin{array}{c}
n+k-1 \\
k-1
\end{array}\right) \cdot x^{n}=\frac{1}{(1-x)^{\frac{k}{2}}} \cdot \frac{1}{(1-x)^{\frac{k}{2}}} \\
& =\sum_{n=0}^{\infty}\left(\sum_{i=0}^{n} \frac{<\frac{k}{2}>_{i}}{i !} \cdot \frac{<\frac{k}{2}>_{n-i}}{(n-i) !}\right) \cdot x^{n}
\end{aligned}
$$

or

$$
\left(\begin{array}{c}
n+k-1 \\
k-1
\end{array}\right)=\sum_{i=0}^{n} \frac{<\frac{k}{2}>_{i}}{i !} \cdot \frac{<\frac{k}{2}>_{n-i}}{(n-i) !}
$$

Combining (5) and (6), there will be an estimation formula immediately deduced

$$
\begin{aligned}
& \left|\sum_{a_{1}+a_{2}+\cdots+a_{k}=n} \sum_{a=1}^{q} \chi(a) P_{a_{1}}\left(\cos \frac{2 \pi a}{q}\right) \cdot P_{a_{2}}\left(\cos \frac{2 \pi a}{q}\right) \cdots P_{a_{k}}\left(\cos \frac{2 \pi a}{q}\right)\right| \\
& =\frac{\sqrt{q}}{2} \cdot\left|\sum_{i=0}^{n} \frac{<\frac{k}{2}>_{i}}{i !} \cdot \frac{<\frac{k}{2}>_{n-i}}{(n-i) !} \cdot[\bar{\chi}(n-2 i)+\bar{\chi}(-(n-2 i))]\right| \\
& \leq \sqrt{q} \cdot \sum_{i=0}^{n} \frac{<\frac{k}{2}>_{i}}{i !} \cdot \frac{<\frac{k}{2}>_{n-i}}{(n-i) !}=\sqrt{q} \cdot\left(\begin{array}{c}
n+k-1 \\
k-1
\end{array}\right) .
\end{aligned}
$$

Theorem 2 is proven completely.

Proof of Theorem 3. We prove Theorem 3 below. From the orthogonality of Chebyshev polynomials of the first kind we know that

$$
\int_{-1}^{1} \frac{T_{m}(x) T_{n}(x)}{\sqrt{1-x^{2}}} d x= \begin{cases}0, & \text { if } m \neq n \\ \frac{\pi}{2}, & \text { if } m=n>0 \\ \pi, & \text { if } m=n=0\end{cases}
$$

If integer $n \geq 1$ with $2 \nmid n$, then for any integer $0 \leq i \leq n$, we have $n-2 i \neq 0$, note that $T_{n}(x)=T_{-n}(x)$, so from (7) and Theorem 1 we have 


$$
\begin{aligned}
& \int_{-1}^{1} \frac{1}{\sqrt{1-x^{2}}}\left(\sum_{a_{1}+a_{2}+\cdots+a_{k}=n} P_{a_{1}}(x) \cdot P_{a_{2}}(x) \cdots P_{a_{k}}(x)\right)^{2} d x \\
& =\int_{-1}^{1} \frac{1}{\sqrt{1-x^{2}}}\left(\sum_{i=0}^{n} \frac{<\frac{k}{2}>_{i}}{i !} \cdot \frac{<\frac{k}{2}>_{n-i}}{(n-i) !} \cdot T_{n-2 i}(x)\right)^{2} d x \\
& =4 \int_{-1}^{1} \frac{1}{\sqrt{1-x^{2}}}\left(\sum_{i=0}^{\left[\frac{n}{2}\right]} \frac{<\frac{k}{2}>_{i}}{i !} \cdot \frac{<\frac{k}{2}>_{n-i}}{(n-i) !} \cdot T_{n-2 i}(x)\right)^{2} d x \\
& =2 \pi \cdot \sum_{i=0}^{\left[\frac{n}{2}\right]}\left(\frac{<\frac{k}{2}>_{i}}{i !} \cdot \frac{<\frac{k}{2}>_{n-i}}{(n-i) !}\right)^{2} .
\end{aligned}
$$

For $n=2 m$, if $n-2 i=0$, then $i=m$. So from (7), Theorem 1 and the methods of proving (8) we have

$$
\begin{aligned}
& \int_{-1}^{1} \frac{1}{\sqrt{1-x^{2}}}\left(\sum_{a_{1}+a_{2}+\cdots+a_{k}=n} P_{a_{1}}(x) \cdot P_{a_{2}}(x) \cdots P_{a_{k}}(x)\right)^{2} d x \\
& =\int_{-1}^{1} \frac{1}{\sqrt{1-x^{2}}}\left(\left(\frac{<\frac{k}{2}>_{m}}{m !}\right)^{2}+2 \sum_{i=0}^{m-1} \frac{<\frac{k}{2}>_{i}}{i !} \cdot \frac{<\frac{k}{2}>_{2 m-i}}{(2 m-i) !} \cdot T_{2 m-2 i}(x)\right)^{2} d x \\
& =\pi \cdot\left(\frac{<\frac{k}{2}>_{m}}{m !}\right)^{4}+2 \pi \cdot \sum_{i=0}^{m-1}\left(\frac{<\frac{k}{2}>_{i}}{i !} \cdot \frac{<\frac{k}{2}>_{n-i}}{(n-i) !}\right)^{2} \\
& =2 \pi \cdot \sum_{i=0}^{m}\left(\frac{<\frac{k}{2}>_{i}}{i !} \cdot \frac{<\frac{k}{2}>_{2 m-i}}{(2 m-i) !}\right)^{2}-\pi \cdot\left(\frac{<\frac{k}{2}>_{m}}{m !}\right)^{4} .
\end{aligned}
$$

Let $x=\sin \theta$, then we have

$$
\begin{aligned}
& \int_{-1}^{1} \frac{1}{\sqrt{1-x^{2}}}\left(\sum_{a_{1}+a_{2}+\cdots+a_{k}=n} P_{a_{1}}(x) \cdot P_{a_{2}}(x) \cdots P_{a_{k}}(x)\right)^{2} d x \\
& =\int_{-\frac{\pi}{2}}^{\frac{\pi}{2}}\left(\sum_{a_{1}+a_{2}+\cdots+a_{k}=n} P_{a_{1}}(\sin \theta) \cdot P_{a_{2}}(\sin \theta) \cdots P_{a_{k}}(\sin \theta)\right)^{2} d \theta .
\end{aligned}
$$

Now Theorem 3 follows from (8), (9), and (10).

\section{Conclusions}

Three theorems and three inferences are the main results in the paper. Theorem 1 gives proof of the symmetry of Legendre polynomials and the symmetry relationship with Chebyshev polynomials of the first kind. This conclusion also improves the early results in [4], and also gives us a different representation for the result in [3]. Theorem 2 obtained an inequality involving Dirichlet characters and Legendre polynomials; this is actually a new contribution to the study of Legendre polynomials and character sums mod $q$. Theorem 3 established an integral identity involving the symmetry sums of the Legendre polynomials. The three corollaries are some special cases of our three theorems for $k=1$, and can not only enrich the research content of the Legendre polynomials, but also promote its research development.

Author Contributions: All authors have equally contributed to this work. All authors read and approved the final manuscript.

Funding: This work is supported by the Y. S. T. N. S. P (2019KJXX-076) and N. S. B. R. P in Shaanxi Province (2019JM-207). 
Acknowledgments: The authors would like to thank the editor and referee for their very helpful and detailed comments, which have significantly improved the presentation of this paper.

Conflicts of Interest: The authors declare that there are no conflicts of interest regarding the publication of this paper.

\section{References}

1. Borwein, P.; Erdèlyi, T. Polynomials and Polynomial Inequalities; Springer: New York, NY, USA, 1995.

2. Jackson, D. Fourier Series and Orthogonal Polynomials; Dover Publications: Mineola, NY, USA, 2004.

3. Shen, S.M.; Chen, L. Some types of identities involving the Legendre polynomials. Mathematics 2019, 7, 114. [CrossRef]

4. Zhou, Y.L.; Wang, X. The relationship of Legendre polynomials and Chebyshev polynomials. Pure Appl. Math. 1999, 15, 75-81.

5. Zhang, Y.X.; Chen, Z.Y. A new identity involving the Chebyshev polynomials. Mathematics 2018, 6, 244. [CrossRef]

6. Kim, T.; Kim, D.S.; Dolgy, D.V. Sums of finite products of Legendre and Laguerre polynomials. Adv. Differ. Equ. 2018, 2018, 277. [CrossRef]

7. Wang, S.Y. Some new identities of Chebyshev polynomials and their applications. Adv. Differ. Equ. 2015, $2015,355$.

8. He, Y. Some results for sums of products of Chebyshev and Legendre polynomials. Adv. Differ. Equ. 2019, 2019, 357. [CrossRef]

9. Chen, L.; Zhang, W.P. Chebyshev polynomials and their some interesting applications. Adv. Differ. Equ. 2017, 2017, 303.

10. Li, X.X. Some identities involving Chebyshev polynomials. Math. Probl. Eng. 2015, 2015, 950695. [CrossRef]

11. Wang, T.T.; Zhang, H. Some identities involving the derivative of the first kind Chebyshev polynomials. Math. Probl. Eng. 2015, 2015, 146313. [CrossRef]

12. Zhang, W.P.; Wang, T.T. Two identities involving the integral of the first kind Chebyshev polynomials. Bull. Math. Soc. Sci. Math. Roum. 2017, 108, 91-98.

13. Kim, T.; Dolgy, D.V.; Kim, D.S. Representing sums of finite products of Chebyshev polynomials of the second kind and Fibonacci polynomials in terms of Chebyshev polynomials. Adv. Stud. Contemp. Math. 2018, 28, 321-336.

14. Cesarano, C. Identities and generating functions on Chebyshev polynomials. Georgian Math. J. 2012, 19, 427-440. [CrossRef]

15. Cesarano, C. Generalized Chebyshev polynomials. Hacet. J. Math. Stat. 2014, 43, 731-740.

16. Dattoli, G.; Srivastava, H.M.; Cesarano, C. The Laguerre and Legendre polynomials from an operational point of view. Appl. Math. Comput. 2001, 124, 117-127. [CrossRef]

17. Islam, S.; Hossain, B. Numerical solutions of eighth order BVP by the Galerkin residual technique with Bernstein and Legendre polynomials. Appl. Math. Comput. 2015, 261, 48-59. [CrossRef]

18. Khalil, H.; Rahmat, A.K. A new method based on Legendre polynomials for solutions of the fractional two-dimensional heat conduction equation. Comput. Math. Appl. 2014, 67, 1938-1953. [CrossRef]

19. Wan, J.; Zudilin, W. Generating functions of Legendre polynomials: A tribute to Fred Brafman. J. Approx. Theory 2013, 170, 198-213. [CrossRef]

20. Nemati, S.; Lima, P. M.; Ordokhani, Y. Numerical solution of a class of two-dimensional nonlinear Volterra integral equations using Legendre polynomials. J. Comput. Appl. Math. 2013, 242, 53-69. [CrossRef]

(C) 2019 by the authors. Licensee MDPI, Basel, Switzerland. This article is an open access article distributed under the terms and conditions of the Creative Commons Attribution (CC BY) license (http:/ / creativecommons.org/licenses/by/4.0/). 